\title{
OPEN Assessing the appropriateness of the management of gastro-oesophageal reflux in Australian children: a population-based sample survey
}

Gaston Arnolda ${ }^{1 凶}$, Harriet Hiscock ${ }^{2,3,4}$, David Moore ${ }^{5}$, Glen Farrow ${ }^{6}$, Peter D. Hibbert ${ }^{1,7}$, Louise K. Wiles ${ }^{1,7}$, Hseun P. Ting ${ }^{1}$, Charlotte J. Molloy ${ }^{1,7}$, Meagan Warwick ${ }^{1}$ \& Jeffrey Braithwaite ${ }^{1}$

Gastro-oesophageal reflux (GOR) is a common physiological state in infants and young children, with gastro-oesophageal reflux disease (GORD) its pathological manifestation. Management of GOR/GORD requires elimination of possible underlying causes, parental reassurance, modification of feeding and symptom mitigation, monitoring, and referral to paediatricians if warning signs are present. Published clinical practice guidelines (CPGs) seek to support clinicians and improve management. This study aimed to measure the proportion of Australian GOR/GORD paediatric care that was in line with CPG recommendations. National and international CPGs for GOR/GORD were systematically identified and candidate indicators extracted; a Delphi process selected 32 indicators relevant to Australian paediatric care in 2012 and 2013. Medical records were identified in General Practices, the offices of general paediatricians, Emergency Departments and inpatient settings. Adherence to indicators was assessed by nine trained paediatric nurses undertaking retrospective medical record review. Medical records were reviewed in 115 healthcare sites; identifying 285 children, three-quarters aged < 1 year, who had 359 visits for management of GOR/GORD; 2250 eligible indicator assessments were performed. Estimated adherence rates are reported for 21 indicators with $\geq 25$ assessments. Five indicators recommending differential diagnostic tests (e.g., urinalysis) for infants presenting with recurrent regurgitation and poor weight gain had $10 \%$ adherence; conversely, avoidance of unrecommended tests (e.g., barium swallow and meal) was high (99.8\% adherence: $95 \% \mathrm{Cl} 97.0-100)$. Avoidance of prescription of acid-suppression medication for infants at the first presentation was higher if they were healthy and thriving (86.9\% adherence: $95 \% \mathrm{Cl} 86.0-96.8)$, intermediate if they had feeding refusal $(73.1 \%$ : $95 \% \mathrm{Cl} 56.0-86.3)$ and lower if they presented with irritability and unexplained crying (58.8\%: 95\% Cl 28.2-85.0). A guideline targeting Australian health professionals caring for infants and children with GOR/GORD is warranted, highlighting the importance of differential diagnostic testing and avoidance of acid-suppression medication in infants.

Abbreviations

CPGs

ED
Clinical practice guidelines

Emergency department

${ }^{1}$ Centre for Healthcare Resilience and Implementation Science, Australian Institute of Health Innovation, Macquarie University, Level 6, 75 Talavera Road, Sydney, NSW 2109, Australia. ${ }^{2}$ Population Health Theme, Murdoch Children's Research Institute, Royal Children's Hospital, Flemington Road, Parkville, VIC 3052, Australia. ${ }^{3}$ Department of Paediatrics, The University of Melbourne, Melbourne, VIC 3010, Australia. ${ }^{4}$ School of Population and Global Health, The University of Melbourne, Melbourne, VIC 3010, Australia. ${ }^{5}$ Women's and Children's Hospital, 72 King William Road, North Adelaide, SA 5006, Australia. 'Sydney Children's Hospital Network, Prince of Wales Hospital, High Street, Randwick, NSW 2031, Australia. ${ }^{7}$ Centre for Population Health Research, School of Health Sciences, University of South Australia, 101 Currie Street, Adelaide, SA 5001, Australia. ${ }^{\circledR}$ email: gaston.arnolda@mq.edu.au 
ESPGHAN/NASPHAN European and North American Societies for Pediatric Gastroenterology, Hepatology and Nutrition

GOR Gastro-oesophageal reflux

GORD Gastro-oesophageal reflux disease

GP General practitioner

NICE UK National Institute for Clinical Excellence

PPIs Proton pump inhibitors

Gastro-oesophageal reflux (GOR) is the passage of gastric contents into the oesophagus, with or without regurgitation or vomiting. When GOR causes troublesome symptoms or complications, it can be diagnosed as gastrooesophageal reflux disease (GORD) ${ }^{1}$. GOR is a normal and common physiological state in infants, peaking at 1-3 months of age and reducing in prevalence to 12 months $^{2,3}$, and GORD a pathological state.

In the US, data from an administrative claims database estimated an incidence of diagnosis of GORD of $12.3 \%$ in children aged $0-1$ years in 2005 (rising sharply from $3.4 \%$ in 2000 ), and about $1 \%$ in older children and adolescents ${ }^{4}$. A 2008 study of French children attending general practitioners (GPs) and paediatricians estimated GORD prevalence of $12.6 \%$ for children aged $0-1$ years, $4.1 \%$ for children aged $2-11$ years, and $7.6 \%$ for adolescents aged 12-17 years ${ }^{5}$. Estimates of the incidence or prevalence of GORD have not been published for Australian children.

Diagnosis of GORD in infants and children is difficult as symptoms cannot be reliably elicited, signs are non-specific, and simple and reliable diagnostic tests are not available. Management therefore requires the elimination of other possible causes and careful monitoring, symptom mitigation, and investigation or referral to specialists if warning signs are present ${ }^{1}$. Treatments include parental reassurance, modification of feeding and pharmaceutical treatment with acid-suppressing therapy ${ }^{6}$. There are significant concerns about inappropriate treatment, particularly with proton pump inhibitors (PPIs) ${ }^{7}$.

International clinical practice guidelines (CPGs) for the treatment of GOR/GORD were developed jointly by the European and North American Societies for Pediatric Gastroenterology, Hepatology and Nutrition (ESPGHAN/NASPHAN) in 2009 ${ }^{1}$. A survey of European paediatricians in 2011-2012 found low compliance with the international CPGs; for example, $82 \%$ reported prescribing PPIs inappropriately ${ }^{8}$. After a randomly selected subset of participating paediatricians was trained in the guidelines, the over-prescription rate reduced to $29 \%{ }^{9}$.

CareTrack Kids (CTK) assessed care of Australian children aged 0-15 years, in 2012 and 2013, to determine the proportion that received care in line with CPG recommendations for 17 common conditions: averaged across the targeted conditions, adherence was estimated to be $59.8 \%$ (95\% CI 57.5-62.0), and at 52.8\% (95\% CI 45.7-59.9) for GOR/GORD overall ${ }^{10}$. This paper presents and discusses the CareTrack Kids results for GOR/ GORD, at indicator level.

\section{Methods}

The CTK methods have been described in detail elsewhere ${ }^{10-12}$. Key aspects, specifically relevant to GOR/GORD, are described below.

Development of indicators. The RAND-UCLA method was modified and applied to develop indicators ${ }^{13}$. A systematic search for Australian and international CPGs for GOR/GORD, relevant for the years 2012-2013, yielded eight CPGs, from which 44 recommendations were extracted. Recommendations were screened and eight were excluded: seven due to indeterminate wording (e.g., actions 'may' or 'could' have been performed) and one guiding statement without a recommended action.

Proposed recommendations were ratified by experts over a two-stage multi-round modified Delphi process, which comprised an email-based three-round internal review and a two-round external review, using a custom-designed wiki ${ }^{12}$. Four clinicians were recruited for the internal $(n=3$; two paediatricians and a GP) and external reviews $(n=1 \text {; a paediatrician })^{14}$. All reviewers completed their assignments independently to minimise group-think ${ }^{15}$.

In the internal review, reviewers recruited by the CTK research team scored each recommendation against three criteria (acceptability, feasibility and impact) ${ }^{12}$ and advised inclusion or exclusion. For the external review, experts were recruited through advertising to the professional colleges; volunteers registered to an online wiki and self-nominated for CTK conditions based on their experience ${ }^{14}$. The external reviewer applied the same scoring criteria as internal reviewers and, in addition, used a nine-point Likert scale to score each recommendation as representative of appropriate care delivered to children during 2012 and $2013^{12,13}$. The clinical lead for each condition commented on reviewers' responses, and made final decisions regarding the inclusion of the recommendations. A total of 15 final recommendations were ratified by experts and formatted into 32 medical record audit indicator questions. All indicator questions are shown, with additional details, in eTable 1, Appendix 1.

Sample size, sampling process and data collection. CTK targeted 400 medical records for GOR/ GORD and 6000 medical records for 16 other conditions. If any of the 6400 medical records targeted and sampled contained an occasion of care for GOR/GORD, the visit was assessed. Detail on the general sampling methods are provided in the main paper ${ }^{10}$; additional details specific to GOR/GORD can be found in in Appendix 2. Briefly, four healthcare settings were sampled: hospital inpatients and Emergency Department (ED) presentations, and consultations with GPs and private-practice paediatricians, in selected health districts in the Australian states of Queensland, New South Wales and South Australia, for children aged $\leq 15$ years receiving care in 2012 and 2013. The overall recruitment rate was $92 \%$ for hospitals and estimated to be $25 \%$ for paediatricians 


\begin{tabular}{|l|l|}
\hline Characteristic & Children in the CTK study N=285 \\
\hline Age $^{\text {a }}-$ no. $(\%)$ \\
\hline$<1$ year & $225(78.9)$ \\
\hline $1-2$ years & $19(6.7)$ \\
\hline $3-4$ years & $2(0.7)$ \\
\hline $5-11$ years & $19(6.7)$ \\
\hline $12-15$ years & $20(7.0)$ \\
\hline Male-no. $(\%)$ & $156(54.7)$ \\
\hline
\end{tabular}

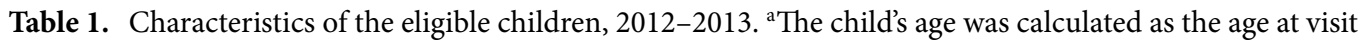
where there was only one, or the midpoint of the child's age at her first and last GORD visit, where there was more than one.

and 24\% for GP (Appendix 2). Data were collected by nine experienced paediatric nurses, trained to assess eligibility for indicator assessment and compliance with CPGs.

Analysis. At indicator level, estimates of compliance were measured as the percentage of eligible indicators which scored as adherent. Weights were constructed to adjust of oversampling of some states, settings and sites ${ }^{10}$. The weighted data were analysed in SAS v9.4 (SAS Institute, Cary NC, USA), using the SURVEYFREQ procedure. Variance was estimated by Taylor series linearization. State and healthcare setting were specified as strata, with the primary sampling unit (health district) specified as the clustering unit. Exact $95 \%$ CIs were generated using the modified Clopper-Pearson method. Results were suppressed if there were $<25$ eligible visits.

Ethical considerations. Primary ethics approval was received from the New South Wales Sydney Children's Hospital Network Ethics Committee (HREC/14/SCHN/113), the Queensland Government Human Research Ethics Committee (HREC/14/QRCH/91), the South Australian Women's and Children's Health Network Ethics Committee (HREC/14/WCHN/68) and the Royal Australian College of General Practitioners Ethics Committee (NREEC 14-008), and site-specific approvals from 34 hospitals. Informed consent was not sought from patients; Australian human research ethics committees can waive requirements for patient consent for external access to medical records if the study entails minimal risk to facilities, clinicians, and patients, and all four abovementioned committees provided this waiver. Informed written consent was received from all participating hospitals and paediatrician and general practitioner practices. Participating health care providers were protected from litigation by gaining statutory immunity for this study as a quality assurance activity from the Federal Minister for Health under Part VC of the Australian Health Insurance Act 1973. All methods were carried out in accordance with relevant guidelines and regulations.

\section{Results}

Details of the 285 children with one or more eligible assessments of CPG adherence for GOR/GORD are provided in Table 1. Seventy-nine percent of the children in the CTK sample were aged under one year, with slightly more males (55\%) than females. Each child had between one and four occasions of care for GOR/GORD which were eligible for assessment (median $=1)$.

Of 12,480 possible indicator assessments, 3648 (29.2\%) were filtered out by age and healthcare setting restrictions and $6582(52.7 \%)$ were designated as not applicable by surveyors. The field team conducted 2250 eligible indicator assessments during 359 visits, at a median of 6 indicators per visit. Eligible GORD visits were assessed in 32 GP and 14 SP practices, 33 hospital EDs and 26 hospital inpatient sites. The distribution of visits by state and provider is summarised in Fig. 1.

Adherence. Estimated adherence for 21 of the 32 indicators is shown in Table 2; 11 indicators are not reported because they had $<25$ visits. For the 21 indicators where adherence was assessed, compliance ranged from $8.5 \%$ for indicator GORD10 ("Infants [...] with recurrent regurgitation and poor weight gain despite adequate energy intake had their blood urea nitrogen assessed") to $99.8 \%$ for GORD14 ("Infants/children who presented with uncomplicated recurrent regurgitation did not have a barium swallow and meal"), the latter being the percentage avoiding an unrecommended diagnostic test. The interquartile range for adherence in the 21 indicators reported was $11.6 \%$ to $73.1 \%$.

There was poor recording of weight and height for infants and children who presented with regurgitation (44.4\%; 95\% CI 33.7-55.5; GORD01), but a higher estimated rate (albeit with wider confidence intervals) if they presented with a history of food refusal or regurgitation (67.3\%; 95\% CI 32.4-91.9; GORD04). There was poor recording of allergies, food and milk intolerances (37.3\%; 95\% CI 19.3-58.3; GORD02).

Infants presenting with recurrent regurgitation and poor weight gain despite adequate energy intake ( $\mathrm{n}=29-32$ visits) appeared poorly served, overall. There was good recording of diet history $(87.6 \%$; $95 \% \mathrm{CI}$ 59.2-91.9; GORD06), but poor compliance with guidelines for diagnostic investigations including urinalysis (10.6\%; 95\% CI 2.3-27.7; GORD07), complete blood count and assessment of serum electrolytes (GORD08 and GORD09; each 9.4\%; 95\% CI 1.8-26.2), blood urea nitrogen (8.5\%; 95\% CI 1.4-25.0; GORD10) and serum creatinine $(8.8 \%$; $95 \%$ CI 1.5-25.4; GORD11). Poor compliance with diagnostic testing is also seen in the performance of urine microscopy, culture and sensitivities (11.6\%; 95\% CI 3.5-26.4; GORD05). 


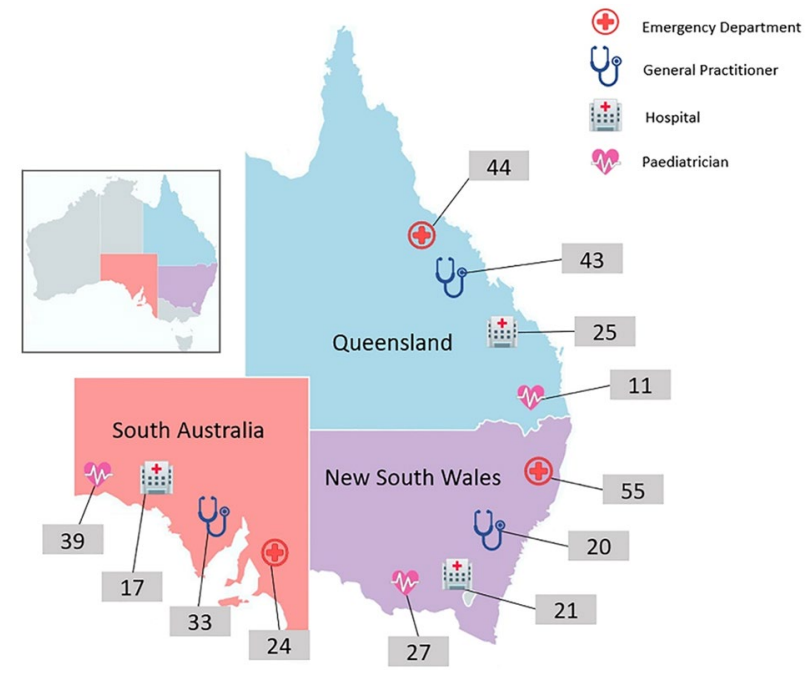

Figure 1. GORD assessments by state and health care provider type. Total number of visits to Emergency Departments $=123$; total number of visits to General Practitioners $=96$; total number of admissions to hospital $=63$; total number of visits to Paediatricians $=77$. Total number of GORD assessments in: New South Wales =123; Queensland $=123$; and South Australia $=113$. Total number of visits assessed for care of GORD in sampling frame $=359$. [Map outline from https://mapchart.net/, Creative Commons Attribution-ShareAlike 4.0 International License, with data added by the authors in Microsoft PowerPoint for Microsoft 365 MSO Version 2102].

Avoiding prescription of acid suppression medications on the first presentation of infants with reflux who were healthy and thriving varied according to other characteristics of the presentation: compliance was highest for infants presenting with frequent regurgitation (86.9\%; 95\% CI 68.0-96.8; GORD19), intermediate for infants presenting with feeding refusal (73.1\%; 95\% CI 56.0-86.3; GORD18) and lowest, but with wide confidence intervals, for infants presenting with irritability or unexplained crying (58.8\%; 95\% CI 28.2-85.0; GORD17).

Three indicators which measure compliance with guidelines to refer children to a paediatric gastroenterologist also had variable results. Estimated compliance was high for infants and children who had weight loss or anorexia or poor feeding (85.9\%; 95\% CI 51.0-99.1; GORD30) and lower, with wider confidence intervals, for those with selected warning signs (55.5\%; 95\% CI 9.4-94.7; GORD28) and for those whose symptoms persisted during and after PPI therapy (49.1\%; 95\% CI 18.3-80.5; GORD31).

\section{Discussion}

A wide range of estimated adherence to guidelines was found for management of GOR/GORD in children. Given the importance of failure to thrive as a warning $\operatorname{sign}^{1}$, it was disappointing that infants and children presenting with regurgitation did not routinely have weight and height recorded. The low level of routine documentation of allergies and food intolerance is similarly concerning.

The ESPGHAN/NASPHAN guidelines conclude that history and physical examination are sufficient for a diagnosis of GORD in older children and adolescents, but not infants and younger children. Compliance was consistently around $10 \%$ for a series of five differential diagnostic tests for infants presenting with recurrent regurgitation with poor weight gain (urinalysis, complete blood count, serum electrolytes, blood urea nitrogen, and serum creatinine), and separately for urine microscopy, culture and sensitivities for infants and children presenting with a history of food refusal or recurrent regurgitation. However, the five diagnostic tests for infants with recurrent regurgitation and poor weight gain are only recommended by the 2009 ESPGHAN/NASPHAN guideline $^{1}$, while the 2018 ESPGHAN/NASPHAN guideline notes that unspecified tailored tests should be undertaken to address differential diagnosis of alarm signs ${ }^{16}$; an Australian guideline notes the role of investigations for differential diagnosis, but does not recommend specific tests ${ }^{6}$.

There was a high rate of referral to a paediatric gastroenterologist (86\%) for infants and children with anorexia, weight loss or poor feeding, but poorer rates of referral for children with warning signs (56\%; see note to Table 2 for full listing of signs) and for children whose symptoms persisted during and after PPI therapy (49\%). By contrast, a 2011 survey of Australian GPs found that $81 \%$ of respondents stated that they refer to a specialist following an ineffective medication trial for $\mathrm{GORD}^{17}$. The difference may reflect a bias in self-reporting, or a lack of affordable access to specialists-in a recent Australian survey, 27\% of GPs noted lack of access to specialists and $22 \%$ noted patient financial status as issues influencing their management of infant GOR/GORD ${ }^{17}$.

Among indicators concerned with overuse of unrecommended treatments, compliance was variable for the prescription of PPIs at the first presentation of a healthy thriving infant, depending on the presentation: $87 \%$ for infants with frequent regurgitation; $73 \%$ for infants with feeding refusal; and $59 \%$ for irritable infants or with unexplained crying, but with wide confidence intervals. A survey of general paediatricians in 11 European countries found that $63 \%$ appropriately avoided prescribing PPIs for infants with recurrent regurgitation and 


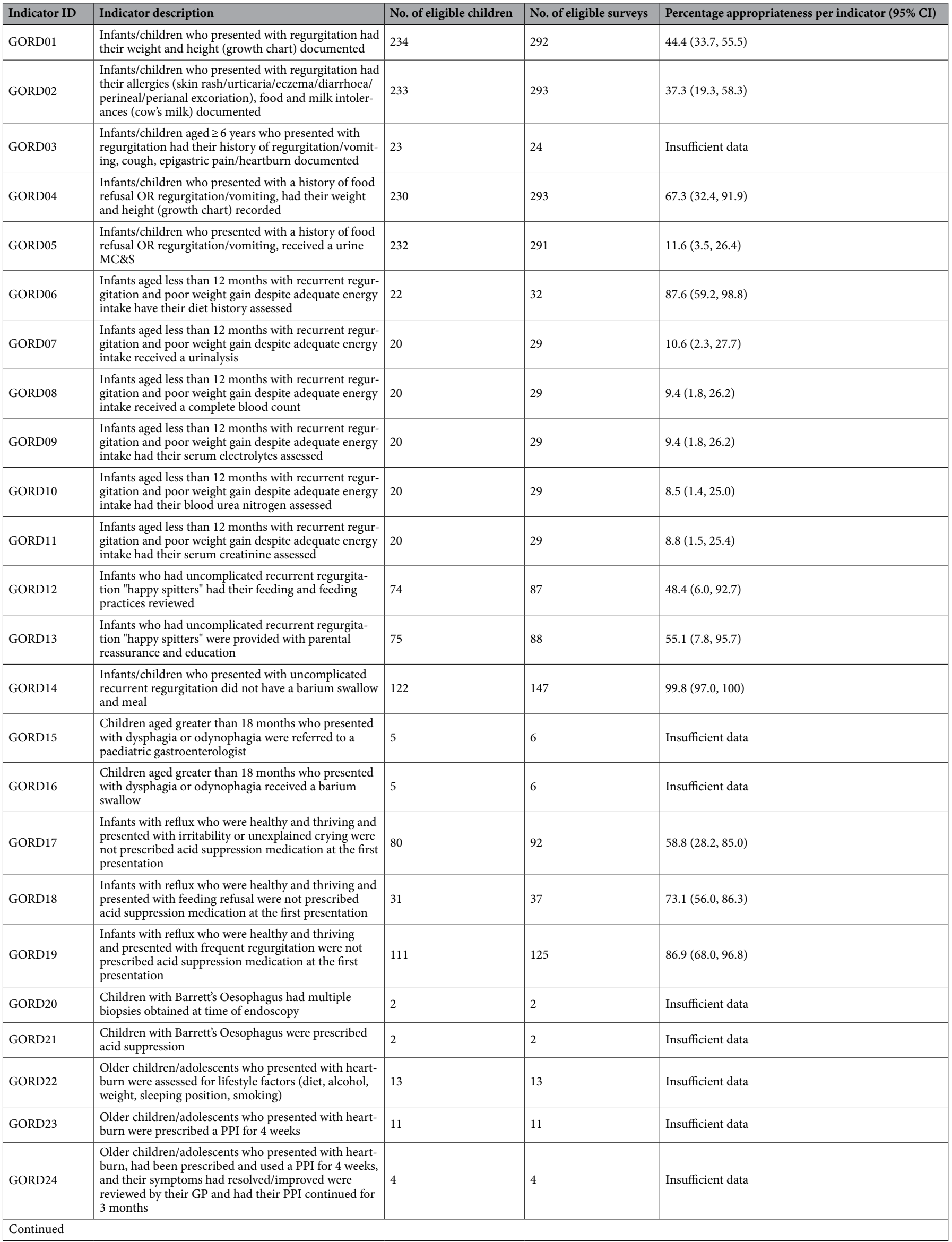




\begin{tabular}{|c|c|c|c|c|}
\hline Indicator ID & Indicator description & No. of eligible children & No. of eligible surveys & Percentage appropriateness per indicator $(95 \% \mathrm{CI})$ \\
\hline GORD25 & $\begin{array}{l}\text { Older children/adolescents who presented with } \\
\text { heartburn, had been prescribed and used a PPI for } \\
4 \text { weeks, and they had recurrent/persistent symptoms } \\
\text { were reviewed by their GP and referred to a gastro- } \\
\text { enterologist }\end{array}$ & 1 & 1 & Insufficient data \\
\hline GORD26 & $\begin{array}{l}\text { Infants/children (aged less than } 18 \text { months) with } \\
\text { reflux oesophagitis had their family lifestyle factors } \\
\text { recorded (diet, alcohol, weight, sleeping position, } \\
\text { smoking) }\end{array}$ & 44 & 55 & $53.1(8.6,93.9)$ \\
\hline GORD27 & $\begin{array}{l}\text { Infants/children (aged less than } 18 \text { months) with } \\
\text { reflux oesophagitis had their symptoms reassessed at } \\
\text { each review }\end{array}$ & 25 & 29 & $80.7(28.1,99.6)$ \\
\hline GORD28 & $\begin{array}{l}\text { Infants/children who had the presence of warning } \\
\text { signs }\left(\text { see definition }{ }^{\mathrm{a}} \text { ) were referred to a paediatric }\right. \\
\text { gastroenterologist }\end{array}$ & 42 & 57 & $55.5(9.7,94.7)$ \\
\hline GORD29 & $\begin{array}{l}\text { Infants/children who had difficulty swallowing or a } \\
\text { history of obstruction were referred to a paediatric } \\
\text { gastroenterologist }\end{array}$ & 14 & 16 & Insufficient data \\
\hline GORD30 & $\begin{array}{l}\text { Infants/children who had weight loss/anorexia/poor } \\
\text { feeding were referred to a paediatric gastroenterologist }\end{array}$ & 42 & 57 & $85.9(51.0,99.1)$ \\
\hline GORD31 & $\begin{array}{l}\text { Infants/children whose symptoms persisted during } \\
\text { and after PPI therapy were referred to a paediatric } \\
\text { gastroenterologist }\end{array}$ & 37 & 45 & $49.1(18.3,80.5)$ \\
\hline GORD32 & $\begin{array}{l}\text { Infants/children with uncomplicated recurrent regur- } \\
\text { gitation who presented with projectile vomiting OR } \\
\text { haematemesis OR bile-stained vomiting, were imme- } \\
\text { diately referred to a hospital emergency department }\end{array}$ & 0 & 0 & Insufficient data \\
\hline
\end{tabular}

Table 2. Appropriateness of care, by clinical indicator. MC\&S Microscopy, Culture and Sensitivities, PPI Proton-Pump Inhibitor. ${ }^{a}$ Includes: Bilious vomiting; Gastrointestinal bleeding; Hematemesis; Hematochezia; Consistently forceful vomiting; Onset of vomiting after 6 months of life; Failure to thrive; Diarrhea; Constipation; Fever; Lethargy; Hepatosplenomegaly; Bulging fontanelle; Macro/microcephaly; Seizures; Abdominal tenderness or distension; Documented or suspected genetic/metabolic syndrome.

55\% avoided prescribed PPIs for unexplained crying or distress; a survey of Italian paediatricians found rates of appropriate avoidance of PPI prescription of $62 \%$ and $44 \%$, respectively; and a 2002-2004 survey of US Family Practitioners found self-reported rates of avoidance of empirical trials of acid suppression of $70 \%$ and $38 \%$, respectively. It is important to note that the European, Italian and US estimates are based on clinicians' responses to case scenarios, while the CTK estimates focus on the first presentation of a child, excluding subsequent consultations. That said, the estimated performance of Australian clinicians appears higher in relation to children presenting with uncomplicated recurrent regurgitation, but broadly similar in relation to infants with unexplained distress. The management of these infants is of concern, as a recent meta-analysis of five randomised controlled trials on the use of PPIs on crying and irritable infants found no evidence of benefit ${ }^{18}$; only one trial reported adverse events, and this found a statistically significant increase in serious adverse events, associated with a higher rate of lower respiratory tract infections ${ }^{19}$.

Australian data on the use of prescription medicines as first line therapy in infants with GORD is limited. A survey of GPs found that $16 \%$ would 'usually' use prescription medicines as first line therapy for GORD, with an additional $56 \%$ doing so 'occasionally'; details were not reported by type of medicine or clinical presentation, but it is notable that a majority of GPs consider PPIs to be highly effective ${ }^{17}$. Studies in the US ${ }^{4}$ and in Belgium ${ }^{20}$ have found marked increases in the use of PPIs for paediatric patients, with concerns about misuse, particularly in infants and younger children.

The authors are aware of one interventional study that sought to improve compliance with CPGs for management of GOR/GORD in children. Baseline data were taken from a 2011-2012 random survey of general paediatricians in 11 European countries, with respondents (42\%) providing information about their diagnosis and treatment for 12 clinical scenarios ${ }^{8}$, to assess adherence to the ESPGHAN/NASPHAN guideline ${ }^{1}$. A followup study trained the first 100 respondents, from a randomly selected listing, who agreed to participate in an intervention and to record details on the management of each child managed for GOR/GORD in the three months following 9 . Two interventions were assessed: a 30-min podcast and set of slides vs a written synopsis of the guidelines; follow-up data was aggregated as no differences were noted between the intervention groups. Results of the data audit for the 100 participants post-intervention were compared to their baseline case scenario responses. The impact of intervention on self-reported practice was marked: $37.1 \%$ of paediatricians stated that they would use a PPI for an infant with recurrent regurgitation in response to the pre-intervention case scenario but only $4.5 \%$ recorded use of a PPI for these infants in the post-training audit of practice; the corresponding rates were $45.2 \%$ pre- and $3.7 \%$ post-intervention for PPI use in infants with unexplained crying or distressed behaviour. Whether this pattern would continue in routine practice, unaccompanied by audit, is unknown.

Despite the differential outcome measurement pre- and post-intervention and the possibility of response bias in the post-intervention period, the results suggest that substantial changes in clinical behaviour of general paediatricians might be achieved with minimal intervention. In Australia, an intensive clinical audit and feedback 
program to improve management of GORD in adults attending GPs reported some successes in improving compliance with evidence-based guidelines ${ }^{21}$, but the changes achieved were marginal.

A 2011 survey of Australian GPs about the management of GOR/GORD in infants found that 37\% of respondents were concerned about the lack of guidelines and education relevant to infants ${ }^{17}$. GORD is discussed in a number of current guidelines including: the National Health and Medical Research Council guideline on infant feeding ${ }^{22}$; consensus panel guidelines for cough in children and adults ${ }^{23}$; and on use of infant formulas to treat cows' milk protein allergy ${ }^{24}$. Management guidance specifically addressing paediatric presentations with GOR/ GORD have also been published for Australian $\mathrm{GPs}^{6}$, and the literature has also been recently reviewed for health professionals with an interest in breastfeeding ${ }^{25}$. Internationally, comprehensive guidelines that were published jointly by ESPGHAN/NASPHAN in 2009 were used in the current study ${ }^{1}$, updated ${ }^{26}$, and supported by the American Academy of Pediatrics ${ }^{27}$; a detailed literature review and separate guidance was also published by the UK National Institute for Clinical Excellence (NICE) ${ }^{28}$, and the ESPGHAN/NASPHAN guideline was updated in $2018^{16}$. This reflects growing recognition of the need to provide health professionals with guidance on the management of GOR/GORD in infants and children, to reduce unwarranted clinical variation and improve management.

The CTK study has numerous strengths and weaknesses which are described in additional detail elsewhere ${ }^{10}$ and summarised briefly here. The data are drawn from a large survey in three States covering 60\% of Australia's paediatric population. For logistical reasons, coverage was limited to larger hospitals providing $\sim 40 \%$ of all inpatient and ED care. While hospitals had excellent participation rates, the participation rates of GPs and specialist providers is estimated to be $\sim 25 \%$; it is plausible that self-selection has led to over-estimation of compliance. Within healthcare sites, random record selection was externally controlled in both hospital and GP settings, but random record selection could not be standardised in paediatricians consulting rooms, with unknown impacts on estimated compliance. The results of 11 indicators had $<25$ responses and were thus too unreliable to publish; the results of several other indicators had wide confidence intervals. Finally, this study assesses documented practice, and it is plausible that this differs from actual practice; in primary care, it has been estimated lack of documentation can lead to underestimation of compliance by around 10 percentage points ${ }^{29}$.

Gaining ethical and administrative clearance to audit over 6000 thousand medical records in over 100 health facilities, undertaking the audits and reporting on each of the audited clinical conditions has led to a substantial passing of time from the period audited (2012-2013) to the time of publication. Clearly, clinical behaviour may have changed in the interim. Moreover, two international clinical guidelines on paediatric GOR/GORD have been published since 2013, the NICE guideline in $2015^{28}$ and the 2018 update of the ESPGHAN/NASPHAN guideline ${ }^{16}$. While these guidelines have not substantively altered the principles underlying the indicators assessed in 2012-2013, some details have changed. For example, the 2009 ESPGHAN/NASPHAN recommendations for five specific tests for regurgitating infants with poor weight gain has been replaced in the 2018 guideline by advice to undertake unspecified tests appropriate to address differential diagnosis for 'alarm signs'. It is noteworthy, however, that the 2018 guidance is effectively unchanged from 2009 in recommending the avoidance of PPIs for infants who are healthy and thriving, even if distressed, while acknowledging that the parents of distressed infants often place clinicians under intense pressure to provide a 'solution'. The overuse of PPIs in infants has been noted as an important example of irrational prescribing in children ${ }^{30}$, for which solutions must be found.

The results suggest that children and infants with GOR/GORD, and their families, could be better served. The health system needs to provide detailed guidance for clinicians involved in the care of these children, providing standards against which they can benchmark and monitor their performance. Such guidance needs to cover simple assessment, such as charting of weight and height, judicious diagnostic testing for differential and positive diagnosis, and avoidance of unjustified pharmaceutical interventions such as use of PPIs in healthy infants who present with irritability and unexplained crying. In addition to interventions targeting clinician behaviour, the needs and beliefs of parents of children with GOR/GORD, and with unexplained crying, need to be understood as a basis for designing interventions targeting parents. This understanding also open the possibility of targeting the clinician-parent interaction through innovative interventions such as shared decision-making programs, which have been demonstrated to be effective in reducing the unwarranted prescription of antibiotics ${ }^{31}$.

\section{Conclusion}

The study shows poor overall compliance with CPGs in the management of infants and children presenting with GOR/GORD, with some recommended actions showing very poor compliance and others showing extremely high compliance. The results suggest that a guideline targeting Australian health professionals caring for infants and children with GOR/GORD is warranted, with multi-faceted interventions to promulgate guidelines and increase sustained compliance, supported by systems of ongoing monitoring and feedback to individual clinicians.

\section{Data availability}

The datasets used and/or analysed during the current study are available from the corresponding author on reasonable request.

Received: 3 September 2020; Accepted: 25 March 2021

Published online: 08 April 2021

\section{References}

1. Vandenplas, Y. et al. Pediatric gastroesophageal reflux clinical practice guidelines: Joint recommendations of the North American Society for Pediatric Gastroenterology, Hepatology, and Nutrition (NASPGHAN) and the European Society for Pediatric Gastroenterology, Hepatology, and Nutrition (ESPGHAN). J. Pediatr. Gastroenterol. Nutr. 49(4), 498-547 (2009).

2. Martin, A. J. et al. Natural history and familial relationships of infant spilling to 9 years of age. Pediatrics 109(6), 1061-1067 (2002). 
3. Hegar, B. et al. Natural evolution of regurgitation in healthy infants. Acta Pcediatr. 98(7), 1189-1193 (2009).

4. Nelson, S. et al. Pediatric gastroesophageal reflux disease and acid-related conditions: Trends in incidence of diagnosis and acid suppression therapy. J. Med. Econ. 12(4), 348-355 (2009).

5. Martigne, L. et al. Prevalence and management of gastroesophageal reflux disease in children and adolescents: A nationwide cross-sectional observational study. Eur. J. Pediatr. 171(12), 1767-1773 (2012).

6. Allen, K. \& Ho, S. Gastro-oesophageal reflux in children: What's the worry?. Aust. Fam. Physician. 41(5), 268-272 (2012).

7. Stark, C. M. \& Nylund, C. M. Side effects and complications of proton pump inhibitors: A pediatric perspective. J. Pediatr. 168(1), 16-22 (2016).

8. Quitadamo, P. et al. European pediatricians' approach to children with GER symptoms: Survey of the implementation of 2009 NASPGHAN-ESPGHAN guidelines. J. Pediatr. Gastroenterol. Nutr. 58(4), 505-509 (2014).

9. Quitadamo, P. et al. Do pediatricians apply the 2009 NASPGHAN-ESPGHAN guidelines for the diagnosis and management of gastroesophageal reflux after being trained?. J. Pediatr. Gastroenterol. Nutr. 59(3), 356-359 (2014).

10. Braithwaite, J. et al. Quality of health care for children in Australia, 2012-2013. JAMA 319(11), 1113-1124 (2018).

11. Hooper, T. D. et al. CareTrack Kids-part 2. Assessing the appropriateness of the healthcare delivered to Australian children: Study protocol for a retrospective medical record review. BMJ Open 5(4), e007749 (2015).

12. Wiles, L. K. et al. CareTrack Kids-part 1. Assessing the appropriateness of healthcare delivered to Australian children: Study protocol for clinical indicator development. BMJ Open 5(4), e007748 (2015).

13. Fitch, K., Bernstein, S. J., Aguilar, M. D., Burnand, B. \& LaCalle, J. R. The RAND/UCLA Appropriateness Method User's Manual (Rand Corp, 2001).

14. Boulkedid, R., Abdoul, H., Loustau, M., Sibony, O. \& Alberti, C. Using and reporting the Delphi method for selecting healthcare quality indicators: A systematic review. PLoS ONE 6(6), e20476 (2011).

15. Hasson, F. \& Keeney, S. Enhancing rigour in the Delphi technique research. Technol. Forecast. Soc. Change. 78(9), 1695-1704 (2011).

16. Rosen, R. et al. Pediatric gastroesophageal reflux clinical practice guidelines: Joint recommendations of the North American Society for Pediatric Gastroenterology, Hepatology, and Nutrition (NASPGHAN) and the European Society for Pediatric Gastroenterology, Hepatology, and Nutrition (ESPGHAN). J. Pediatr. Gastroenterol. Nutr. 66(3), 516 (2018).

17. Kirby, C. N., Segal, A. Y., Hinds, R., Jones, K. M. \& Piterman, L. Infant gastro-oesophageal reflux disease (GORD): Australian GP attitudes and practices. J. Paediatr. Child Health. 52(1), 47-53 (2016).

18. Gieruszczak-Bialek, D., Konarska, Z., Skorka, A., Vandenplas, Y. \& Szajewska, H. No effect of proton pump inhibitors on crying and irritability in infants: Systematic review of randomized controlled trials. J. Pediatr. 166(3), 767-70.e3 (2015).

19. Orenstein, S. R., Hassall, E., Furmaga-Jablonska, W., Atkinson, S. \& Raanan, M. Multicenter, double-blind, randomized, placebocontrolled trial assessing the efficacy and safety of proton pump inhibitor lansoprazole in infants with symptoms of gastroesophageal reflux disease. J. Pediatr. 154(4), 514-520 (2009).

20. De Bruyne, P., Christiaens, T., Vander Stichele, R. \& Van Winckel, M. Changes in prescription patterns of acid-suppressant medications by Belgian pediatricians: Analysis of the national database [1997-2009]. J. Pediatr. Gastroenterol. Nutr. 58(2), 220-225 (2014).

21. Kirby, C. N., Piterman, L., Nelson, M. \& Dent, J. Gastro-oesophageal reflux disease: Impact of guidelines on GP management. Aust. Fam. Physician. 37(1/2), 73 (2008).

22. National Health and Medical Research Council. Infant Feeding Guidelines: Information for health workers (National Health and Medical Research Council, 2012).

23. Gibson, P. G. et al. CICADA: Cough in children and adults: Diagnosis and assessment. Australian cough guidelines summary statement. Med. J. Aust. 192(5), 265-271 (2010).

24. Kemp, A. S. et al. Guidelines for the use of infant formulas to treat cows milk protein allergy: An Australian consensus panel opinion. Med. J. Aust. 188(2), 109 (2008).

25. Anderson, J. C. Infant reflux-not as simple as we might think. Breastfeeding Rev. 25(2), 25 (2017).

26. Vandenplas, Y. \& Hauser, B. An updated review on gastro-esophageal reflux in pediatrics. Expert Rev. Gastroenterol. Hepatol. 9(12), 1511-1521 (2015).

27. Lightdale, J. R. \& Gremse, D. A. Gastroesophageal reflux: management guidance for the pediatrician. Pediatric 131(5), e168495 (2013).

28. N National Collaborating Centre for Women's Children's Health (UK). Gastro-Oesophageal Reflux Disease: Recognition, Diagnosis and Management in Children and Young People (National Institute for Health and Care Excellence (UK), 2015). https://www.ncbi. nlm.nih.gov/books/NBK293624/

29. McGlynn, E. A. et al. The quality of health care delivered to adults in the United States. N. Engl. J. Med. 348(26), 2635-2645 (2003).

30. Choonara, I. Rational Prescribing is Important in All Settings (2013).

31. Tonkin-Crine, S. K. G. et al. Clinician-targeted interventions to influence antibiotic prescribing behaviour for acute respiratory infections in primary care: an overview of systematic reviews. Cochrane Database Syst. Rev. 2017, 9 (2017).

\section{Acknowledgements}

We acknowledge with gratitude the CareTrack Kids Investigative Team, in particular the fieldwork conducted by our surveying team: Florence Bascombe, Jane Bollen, Samantha King, Naomi Lamberts, Amy Lowe, AnnMarie McEvoy, Stephanie Richardson, Jane Summers, and Annette Sutton; thanks also go to Stan Goldstein, Annie Lau and Nicole Mealing for their earlier contributions.

\section{Author contributions}

J.B. and P.D.H. designed the overall study. J.B., P.D.H., G.A., H.P.T., L.K.W. and C.J.M. carried out the data collection and statistical analysis of the data. H.H., D.M. and G.F. reviewed and made substantial contributions to the earlier drafts. M.W. provided logistics support, assisted in coordination and contributed to the drafting and finalisation of the manuscript. All authors made substantial contributions to the interpretation of results, drafting of the manuscript and writing of the final manuscript. All authors read and approved the final manuscript.

\section{Funding}

The CareTrack Kids study was funded by an Australian National Health and Medical Research partnership Grant (APP1065898), with contributions by the National Health and Medical Research Council, Bupa Health Foundation, Sydney Children's Hospital Network, New South Wales Kids and Families, Children's Health Queensland, and the South Australian Department of Health (SA Health). The funders had no role in the design and conduct of the study; collection, management, analysis, and interpretation of the data; preparation, review, or approval of the manuscript; and decision to submit the manuscript for publication. 


\section{Competing interests}

The authors declare no competing interests.

\section{Additional information}

Supplementary Information The online version contains supplementary material available at https://doi.org/ 10.1038/s41598-021-87369-7.

Correspondence and requests for materials should be addressed to G.A.

Reprints and permissions information is available at www.nature.com/reprints.

Publisher's note Springer Nature remains neutral with regard to jurisdictional claims in published maps and institutional affiliations.

(c) (1) Open Access This article is licensed under a Creative Commons Attribution 4.0 International License, which permits use, sharing, adaptation, distribution and reproduction in any medium or format, as long as you give appropriate credit to the original author(s) and the source, provide a link to the Creative Commons licence, and indicate if changes were made. The images or other third party material in this article are included in the article's Creative Commons licence, unless indicated otherwise in a credit line to the material. If material is not included in the article's Creative Commons licence and your intended use is not permitted by statutory regulation or exceeds the permitted use, you will need to obtain permission directly from the copyright holder. To view a copy of this licence, visit http://creativecommons.org/licenses/by/4.0/.

(C) The Author(s) 2021 